\title{
The advantages of model fitting compared to model simulation in research on preference construction
}

\author{
Edgar Erdfelder*, Marta Castela, Martha Michalkiewicz and Daniel W. Heck \\ Cognition and Individual Differences Lab, Department of Psychology, University of Mannheim, Mannheim, Germany \\ ${ }^{*}$ Correspondence: erdfelder@psychologie.uni-mannheim.de
}

Edited by:

Adele Diederich, Jacobs University Bremen, Germany

Reviewed by:

David Kellen, Albert-Ludwigs-Universität Freiburg, Germany

Keywords: multinomial modeling, model fitting, model simulation, preference construction, serial-position effects, primacy and recency effects

Mantonakis et al. (2009) suggested the pairwise-competition model (PCM) of preference construction. The basic idea is that humans form preferences among choice objects (e.g., types of wines) sequentially, such that the preferred object is the ultimate winner of a sequence of pairwise competitions: The object sampled first is compared to the second, the winner of this competition is then compared to the third object, resulting in a winner that is compared to the fourth, and so on. For a set of $m$ objects, this recursive process terminates after $m$-1 pairwise competitions once the final object has been compared against the previous favorite. Canic and Pachur (2014) recently proposed a formal specification and elaboration of the PCM. Moreover, using computer simulations, they showed how variations in the model's parameters affect primacy and recency effects in sequential choice and how the model can account for various patterns of serial position effects documented in the literature.

We appreciate the contributions of Mantonakis and collaborators as well as Canic and Pachur. Clearly, innovative theoretical ideas and their formal clarification are key elements of progress in science. However, we argue that making use of the full spectrum of formal modeling advantages necessarily involves fitting the model to empirical data. By narrowing down model-based analyses to computer simulations - as Canic and Pachur (2014) did-important questions remain unanswered and misleading conclusions cannot be ruled out.

First, simulating a model and showing that it predicts serial-position effects similar to those found in empirical data does not imply that the model fits these data. A successful model should account for the entire distribution of observed data, not just for selected qualitative aspects such as primacy and recency effects. Formal goodness-of-fit tests provide the necessary information but simulations do not.

Second, if there are several nested versions of a model as in case of the PCM, simulations cannot tell us which model version provides the best account of the data taking model complexity into account (as influenced, among others, by the number of estimated parameters). The model fitting toolbox, in contrast, includes appropriate model selection measures, enabling us to identify the most parsimonious model version that is consistent with the data.

Third, model fitting involves parameter estimation, that is, identification of the parameter values that best account for an existing set of data. It also provides statistical tests for parameters, enabling statistically sound evaluations of parameter differences between groups or conditions. Simulations, in contrast, do not provide such an elaborated framework. Because they ignore sampling variability in empirical data, they may mistakenly suggest parameter differences when in fact there are none.

To illustrate the advantages of model fitting, we apply Canic and Pachur's (2014) formalization of the PCM to the data of Mantonakis and collaborators ${ }^{1}$.

\footnotetext{
${ }^{1}$ We are grateful to Antonia Mantonakis for providing the raw frequencies.
}

\section{THE PCM AS A MULTINOMIAL PROCESSING TREE MODEL}

Fortunately, fitting the PCM to empirical data is straightforward. Inspection of the model equations reveals that the PCM belongs to the class of multinomial processing tree (MPT) models (Batchelder and Riefer, 1999), a model family with a well-developed statistical theory ( $\mathrm{Hu}$ and Batchelder, 1994) that has already been implemented in easy-to-use software tools (Moshagen, 2010; Singmann and Kellen, 2013). MPT models are models for categorical data, such as frequencies $f_{m, j}$ of preferring the $j$-s object in the sequence, $j=1$, $\ldots, m$, given the total number of objects is $m$. They assume a joint multinomial distribution of the frequencies with underlying category probabilities that correspond to sums of products of $S$ model parameters $\theta_{\mathrm{s}}$ and their complements $\left(1-\theta_{\mathrm{s}}\right), s=$ $1, \ldots, S$, each being an element of the unit interval $[0,1]$. The basic MPT model architecture has been found useful to represent sequences of discrete cognitive processes underlying task performance (for a review, see Erdfelder et al., 2009) and, more generally, relations between latent psychological states and categorical test results (e.g., Botella et al., 2013; Erdfelder and Moshagen, 2013).

To formalize the PCM within the MPT framework, we first consider the simplest special case of choosing among $m=2$ objects. According to the PCM, humans show "choice inertia" and stick to their previous favorite (i.e., the first object) with probability $\pi$, thus avoiding any comparison. With the complementary probability $(1-\pi)$, they enter the competition state, in which the second object wins with 
probability $p$ (new) (determined by Luce's choice rule) and, by implication, the first one with probability $1-p$ (new). Thus, preference for the first object can result from choice inertia (probability $\pi$ ) or from the first object winning against the second when choice inertia is absent (probability $(1-\pi)(1-p(n e w)))$. The overall probability of choosing the first object is the sum of these probabilities:

$$
\begin{aligned}
& p(\text { object } 1)= \\
& \pi+(1-\pi) \cdot(1-p(\text { new })) .(1)
\end{aligned}
$$

In contrast, preference for the second object can result from a single sequence only:

$$
p(\text { object } 2)=(1-\pi) \cdot p(\text { new }) .
$$

These equations match the basic MPT structure. Generalization to choice among three or more objects is straightforward because this only involves recursive applications of the processes already described (although not necessarily with the same probabilities). The corresponding model specification file required for analyzing the data of Mantonakis and collaborators using the multiTree program (Moshagen, 2010) can be requested from the first author. It provides for a 2 -factorial design (factor A: Number of wine samples in the choice set: 2-5; factor B: Knowledge about wines: high vs. low) as implemented by Mantonakis et al. (2009).

\section{FITTING THE PCM TO THE DATA OF MANTONAKIS ET AL. (2009)}

Mantonakis et al. (2009) assigned their 142 participants (69 people with high and 73 people with low knowledge about wines) randomly to 4 groups receiving choice sets consisting of 2-5 wine samples, respectively. Following the tasting sequence, each participant indicated her or his preferred wine in the set. Unbeknownst to the participants, the same wine was actually used in each sample. Thus, it seems reasonable to constrain the probabilities $p(\text { new })_{j}, j=$ $2, \ldots, 5$, that the $j$-s wine sample wins the competition against the current favorite to $p(\text { new })_{j}=0.5$ for all participants and choice sets $j$. Our first version of the model, $\operatorname{PCM}(1)$, is based on this assumption only. Consequently, all choice inertia parameters $\pi_{j}, j=2, \ldots, 5$, are left unconstrained for both high and low-knowledge participants. This model accounts for $2 \cdot(1+$ $2+3+4)=20$ independent choice probabilities across the $2 \cdot 4=8$ groups by 8 choice inertia parameters and is identifiable. It fits the observed choice frequencies for wine experts, $G^{2}(6)=5.63$, $p=0.47$, non-experts, $G^{2}(6)=3.97, p=$ 0.68 , and for both samples analyzed conjointly, $G^{2}(12)=9.60, p=0.65$.

Figure 1A displays the maximum likelihood (ML) estimates of the $\pi_{j}$ along with the $95 \%$ confidence intervals. Descriptively, there appears to be an inverted $\mathrm{u}$-shaped trend for experts and a monotonically increasing trend for nonexperts with increasing sequence lengths. However, an equality constraint on the 4 inertia parameters (i.e., $\pi_{2}=\pi_{3}=\pi_{4}=$ $\pi_{5}$ ) does not decrease the fit significantly, neither for experts, $\Delta G^{2}(3)=7.07, p=$ 0.07 , nor for non-experts, $\Delta G^{2}(3)=3.93$, $p=0.27$, nor for both groups analyzed conjointly, $\Delta G^{2}(6)=11.00, \quad p=0.09$. The second model version, $\operatorname{PCM}(2)$, includes both constraints and shows an excellent overall fit, $G^{2}(18)=20.61$, $p=0.30$. Surprisingly, the choice inertia estimate is only slightly lower for highknowledge compared to low-knowledge participants ( 0.485 vs. 0.532 , respectively), a difference that is far from being significant, $\Delta G^{2}(1)=0.21, p=0.64$. This suggests an even more restrictive model version $\operatorname{PCM}(3)$ with all eight inertia parameters equated. With a single remaining choice inertia estimate for both groups $(0.510$, shown as a constant line in Figure 1A), $\operatorname{PCM}(3)$ provides an excellent overall fit, $G^{2}(19)=20.82, p=0.35$.

Given that all three models fit the data nicely it must be expected that the model with the fewest free parameters performs best in terms of model selection criteria that penalize model complexity. Because standard methods such as the Akaike and the Bayesian information criterion (AIC and BIC) focus on the number of free parameters only and ignore differences in the functional form of the models, we used the more sophisticated Fisher information approximation (FIA; Wu et al., 2010). FIA can safely be applied in the present case because the Mantonakis sample exceeds Heck's lower bound for FIA applications (i.e., $N=142>N^{\prime}=16$; see Heck et al., 2014). The FIA values for the models $\operatorname{PCM}(1), \operatorname{PCM}(2)$, and $\operatorname{PCM}(3)$ were 166.4, 165.4, and 163.8, respectively, showing that $\operatorname{PCM}(3)$ performs best. In order to better judge the evidence for each of the three models, we computed the relative weights $w_{\text {FIA }}$ (i.e., estimates of the probabilities of each model being the best one in the set). The FIA weights 0.056 , 0.158 , and 0.786 for the models $\operatorname{PCM}(1)$, $\operatorname{PCM}(2)$, and $\operatorname{PCM}(3)$ highlight that a single inertia parameter suffices to account for the data.

In sum, both goodness-of-fit tests and model selection criteria suggest that $\operatorname{PCM}(3)$ is the best model, despite the fact that this model assumes neither serial position effects for $\pi_{j}$ and $p(\text { new })_{j}$ nor differences in parameters between high- vs. low-knowledge participants. As illustrated in Figures 1B,C, this model accounts for the primacy and recency effects in the preference curves of high and low knowledge participants, respectively, although it includes a single inertia parameter only.

\section{DISCUSSION}

What do we learn from this model fitting exercise? First, we can now say that the PCM indeed fits the entire distribution of preferential choice data obtained by Mantonakis et al. (2009), not just the primacy and recency effects discussed by Canic and Pachur (2014). Somewhat surprisingly, however, the model requires much less parameters to account for order effects in preferential choice data than hypothesized by Mantonakis et al. (2009) and proposed by Canic and Pachur (2014) based on their simulation results. As shown here, there is no evidence for significant differences in choice inertia, neither across the course of the choice sequence nor between high- and low-knowledge participants. Rather, both groups of participants exhibit choice inertia roughly $50 \%$ of the time. Of course, we cannot rule out that this picture would change for groups with larger knowledge differences than in the Mantonakis sample. Moreover, the moderate sample size may play a role: Given a significance level of $\alpha=0.05$ and the current $N=142$, the power of the $G^{2}(1)$ goodness-of-fit test is appropriate for medium deviations from the null hypothesis (i.e., $w=0.3 ; 1-\beta=$ 0.95) but not for small deviations (i.e., 
A Parameter estimates for $\operatorname{PCM}(1)$ and $\operatorname{PCM}(3)$

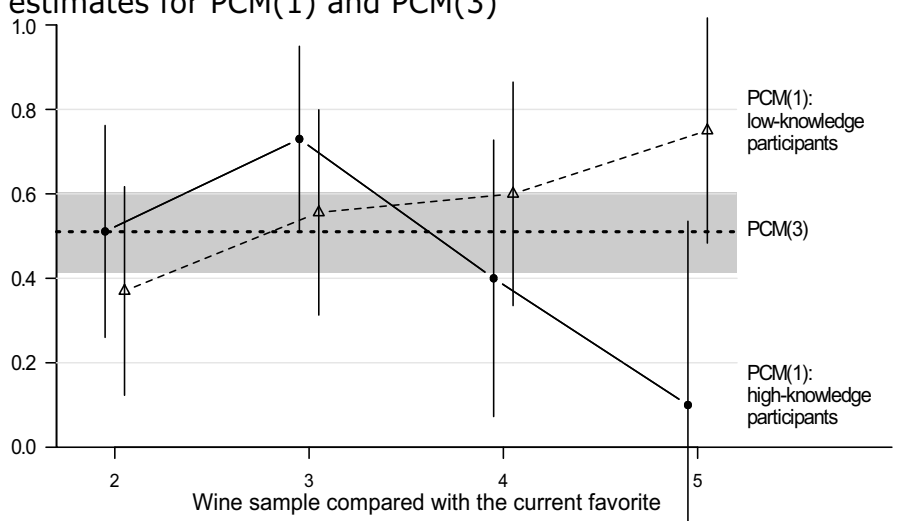

B Observed (solid line) and expected (dashed line) frequencies for high-knowledge participants on PCM(3)
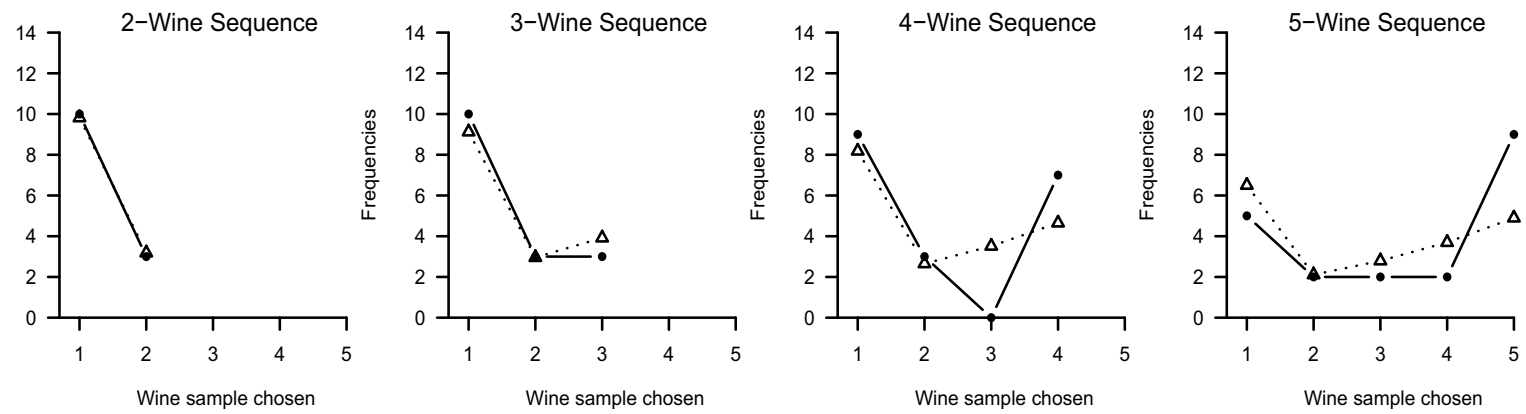

C Observed (solid line) and expected (dashed line) frequencies for low-knowledge participants based on PCM(3)
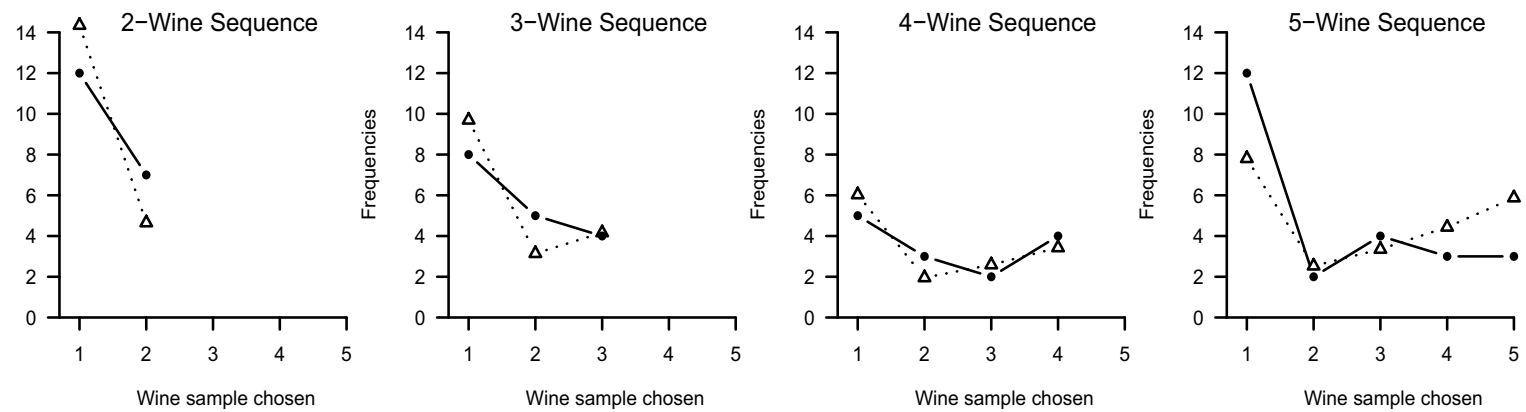

FIGURE 1 | (A) Estimates of the choice inertia parameters of models PCM(1) and PCM(3). Vertical lines indicate 95\% confidence intervals for the eight parameters of PCM(1). The gray area illustrates the $95 \%$ confidence interval of the single $\operatorname{PCM}(3)$ choice inertia parameter. $(\mathbf{B}, \mathbf{C})$
Observed (solid lines) and expected frequencies (dashed lines) for the 1-parameter PCM(3) model applied to the choice frequencies of Mantonakis et al.'s (2009) high vs. low knowledge participants, respectively. $w=0.1 ; 1-\beta=0.22)$. A sample more than five times larger than the Mantonakis sample (specifically, $N=785$ ) would be necessary to achieve a power of 0.80 in the latter case (Faul et al., 2009). Given the present data, however, nothing more can be said than that a single free parameter suffices to fit them well.

Future research should conduct stronger tests of the PCM than presently possible, both by using larger samples and by applying the model not just to the final preferences but to the entire vector of (preliminary) preferences during the tasting sequence. Unfortunately, although it is straightforward to generalize the PCM accordingly, appropriate process data for evaluating such a generalized PCM are currently unavailable. In addition, it would be desirable to generalize the PCM to a multilevel model that captures individual variability in parameters. Again, statistical frameworks for such a generalization are available (e.g., Klauer, 2006, 2010) but appropriate data (including several preference judgments per participant) are still lacking. Last but not least, one might think about comparing the PCM to alternative models of order effects in preference data, for example, models based on signal-detection theory for forced-choice judgments (e.g., DeCarlo, 2012). 
To conclude, given the presently available data of Mantonakis et al. (2009), the up-to-date model fitting procedures that we relied on do not confirm conjectures about sequential trends or knowledge effects in choice inertia parameters as suggested by Canic and Pachur's simulation results. However, they do support the conclusion that Canic and Pachur's formal implementation of the PCM is an empirically successful model of preference formation.

\section{ACKNOWLEDGMENTS}

Manuscript preparation was supported by a grant from the Deutsche Forschungsgemeinschaft (Er 224/2-2). We would like to thank Ann-Kathrin Zaiser for her help in preparing the figures.

\section{REFERENCES}

Batchelder, W. H., and Riefer, D. M. (1999). Theoretical and empirical review of multinomial process tree modeling. Psychon. Bull. Rev. 6, 57-86. doi: 10.3758/BF03210812

Botella, J., Huang, H., and Suero, M. (2013). Multinomial tree models for assessing the status of the reference in studies of the accuracy of tools for binary classification. Front. Psychol. 4:694. doi: 10.3389/fpsyg.2013.00694

Canic, E., and Pachur, T. (2014). Serial-position effects in preference construction: a sensitivity analysis of the pairwise-competition model. Front. Psychol. 5:902. doi: 10.3389/fpsyg.2014. 00902

DeCarlo, L. T. (2012). On a signal detection approach to $m$-alternative forced choice with bias, with maximum likelihood and Bayesian approaches to estimation. J. Math. Psychol. 56, 196-127. doi: 10.1016/j.jmp.2012.02.004

Erdfelder, E., Auer, T.-S., Hilbig, B. E., Aßfalg, A., Moshagen, M., and Nadarevic, L. (2009). Multinomial processing tree models. A review of the literature. Z. Psychol. 217, 108-124. doi: 10.1027/0044-3409.217.3.108

Erdfelder, E., and Moshagen, M. (2013). Conjoint measurement of disorder prevalence, test sensitivity, and test specificity: notes on Botella, Huang, and Suero's multinomial model. Front. Psychol. 4:876. doi: 10.3389/fpsyg.2013.00876

Faul, F., Erdfelder, E., Buchner, A., and Lang, A.-G. (2009). Statistical power analyses using $G^{\star}$ Power 3.1: tests for correlation and regression analyses. Behav. Res. Methods 41, 1149-1160. doi: 10.3758/BRM.41.4.1149

Heck, D. W., Moshagen, M., and Erdfelder, E. (2014). Model selection by minimum description length: lower-bound sample sizes for the Fisher information approximation. J. Math. Psychol. 60, 29-34. doi: 10.1016/j.jmp.2014.06.002

$\mathrm{Hu}, \mathrm{X}$., and Batchelder, W. H. (1994). The statisti$\mathrm{cal}$ analysis of general processing tree models with the EM algorithm. Psychometrika 59, 21-47. doi: 10.1007/BF02294263

Klauer, K. C. (2006). Hierarchical multinomial processing tree models: a latent-class approach. Psychometrika 71, 7-31. doi: 10.1007/s11336-0041188-3

Klauer, K. C. (2010). Hierarchical multinomial processing tree models: a latent-trait approach. Psychometrika 75, 70-98. doi: 10.1007/s11336009-9141-0

Mantonakis, A., Rodero, P., Lesschaeve, I., and Hastie, R. R. (2009). Order in choice: effects of serial position on preferences. Psychol. Sci. 20, 1309-1312. doi: 10.1111/j.1467-9280.2009.02453.x

Moshagen, M. (2010). multiTree: a computer program for the analysis of multinomial processing tree models. Behav. Res. Methods 42, 42-54. doi: 10.3758/BRM.42.1.42

Singmann, H., and Kellen, D. (2013). MPTinR: analysis of multinomial processing tree models in R. Behav. Res. Methods 45, 560-575. doi: 10.3758/s13428-012-0259-0

Wu, H., Myung, J. I., and Batchelder, W. H. (2010). On the minimum description length complexity of multinomial processing tree models. J. Math. Psychol. 54, 291-303. doi: 10.1016/j.jmp.2010.02.001

Conflict of Interest Statement: The reviewer David Kellen declares that, despite having collaborated with authors Marta Castela and Edgar Erdfelder, the review process was handled objectively and no conflict of interest exists. The authors declare that the research was conducted in the absence of any commercial or financial relationships that could be construed as a potential conflict of interest.

Received: 14 November 2014; paper pending published: 08 December 2014; accepted: 27 January 2015; published online: 18 February 2015.

Citation: Erdfelder E, Castela M, Michalkiewicz M and Heck DW (2015) The advantages of model fitting compared to model simulation in research on preference construction. Front. Psychol. 6:140. doi: 10.3389/fpsyg. 2015.00140

This article was submitted to Cognitive Science, a section of the journal Frontiers in Psychology.

Copyright () 2015 Erdfelder, Castela, Michalkiewicz and Heck. This is an open-access article distributed under the terms of the Creative Commons Attribution License (CC BY). The use, distribution or reproduction in other forums is permitted, provided the original author(s) or licensor are credited and that the original publication in this journal is cited, in accordance with accepted academic practice. No use, distribution or reproduction is permitted which does not comply with these terms. 